\title{
Synthesis and characterization of $\mathrm{Bi}_{2} \mathrm{O}_{3}$ nanoparticles by solvothermal method
}

\author{
M. Akhter and M.A.Shah* \\ Special Centre for Nano science, Department of Physics, \\ National Institute of Technology Hazratbal Srinagar-190006 \\ *Email:shah@nitsri.net
}

Bismuth is an advanced functional material with potential applications such as photo catalytic decomposition material, functional electronic material, and optical material, burning rate catalyst, anti-radiative material and medical composite material. We have employed an improved and surfactant free approach for the synthesis of Bismuth oxide $\left(\mathrm{Bi}_{2} \mathrm{O}_{3}\right)$ nanoparticles at very low temperature of $110^{\circ} \mathrm{C}$. This new approach is based on a reaction of bismuth powder and de-ionized (DI) water without the use of any additives or surfactants. The optical, photoelectrical and photoluminescence properties of bismuth oxide films prepared by thermal oxidation of bismuth thin films has been studied [1]. At present, various methods are introduced for the synthesis of the nanoscale $\mathrm{Bi}_{2} \mathrm{O}_{3}$ particles including sol-gel method [2]. Solvothermal synthesis of $\mathrm{Bi}_{2} \mathrm{O}_{3}$ nanoparticles has been achieved in order to control phase structure and morphology [3]. XRD and SEM have been employed to characterize the $\mathrm{Bi}_{2} \mathrm{O}_{3}$ nanoparticles. By the morphological investigations using SEM, it was observed that the grown $\mathrm{Bi}_{2} \mathrm{O}_{3}$ products are having dimensions in the range of $3 \mathrm{~nm}$ to $25 \mathrm{~nm}$. The reported method besides being organics free is economical, fast and free of pollution, which will make it suitable for large scale production.

The formation of $\mathrm{Bi}_{2} \mathrm{O}_{3}$ nanoparticles from the reagents of bismuth and de-ionized water can be explained with the help of facile reaction as follows:

$2 \mathrm{Bi}(\mathrm{s})+3 \mathrm{H}_{2} \mathrm{O}(\mathrm{l}) \rightarrow \mathrm{Bi}_{2} \mathrm{O}_{3}(\mathrm{~s})+3 \mathrm{H}_{2}(\mathrm{~g})$

It is observed that the grown product $\left(\mathrm{Bi}_{2} \mathrm{O}_{3}\right.$ nanoparticles) has different shapes like triangular, rectangular, spherical, etc.

Keywords: nanoparticles, morphological studies, structural studies
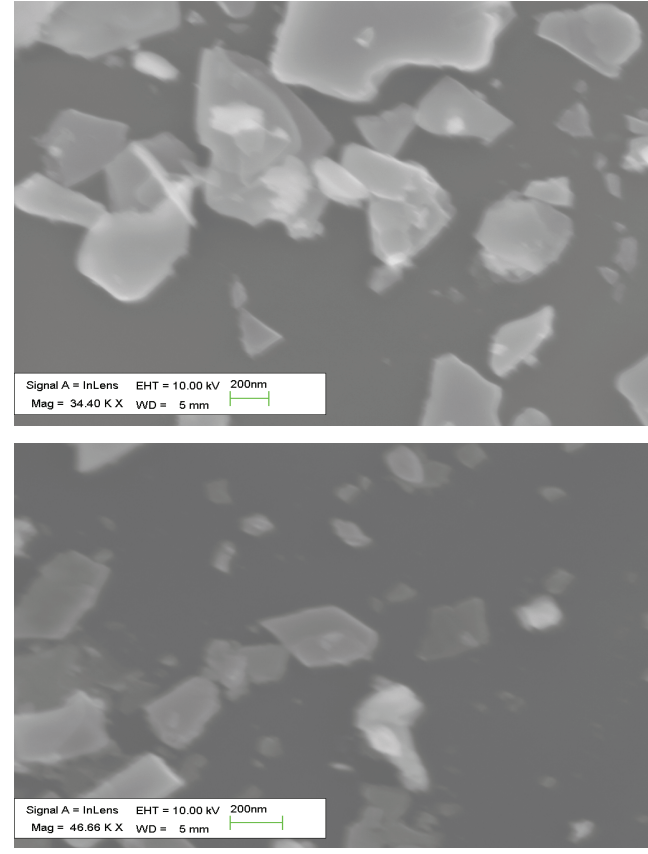

Figure 1: SEM image of $\mathrm{Bi}_{2} \mathrm{O}_{3}$ nanostructures

\section{References}

1. Leontie, L., Caraman, M., Visinoiu, A., \& Rusu, G. I. (2005). On the optical properties of bismuth oxide thin films prepared by pulsed laser deposition. Thin Solid Films, 473(2), 230-235.

2. M. Mallahi1, A. Shokuhfar2, M. R.Vaezi3, A. Esmaeilirad4, V. Mazinani, Synthesis and characterization of Bismuth oxide nanoparticles via sol-gel method, American Journal of Engineering Research, volume 3, pp.162-165, 2014.

3. Luan, X., Jiang, J., Yang, Q., Chen, M., Zhang, M., \& Li, L. (2015). Facile synthesis of bismuth oxidenanoparticles by a hydrolysis solovothermal route and their visible light photo catalytic activity. Environmental Engineering and Management Journal, 14(3), 703-707. 